Veränderuug des Klimas ist, andernfalls gehen diese Patienten rettungslos zu Grunde.

Die Versuche selbst habe ich in der Weise angestellt, dass ich die betreffenden Patienten in das Lazareth aufnehme, sie bei genauer Temperaturbestimmung ohne jedes Medicament liess, bis ich über die Diagnose klar war, genau Eintritt, Dauer und Natur des Anfalls kannte und dann Alumen ustum gab in Dosen à 1,0, so dass die letzte Dosis eine Stunde vor dem vermuthlichen Eintritt des Allfalls genommen wurde. Aeusserte sich die Malariainfection in einer Febris continua, so liess ich das Medicament gewöhnlich am Nachmittag nehmen.

Die Anzahl der einzelnen Dosen, also die Grösse der Tagesgabe, richtete sich nach der Höhe der Temperatur und nach der Dauer des ersten Anfalls, je höher die Temperatur, je länger die Dauer desselben, um so grösser die Tagesgabe. Die grösste Gesammtdosis, die ich pro die gegeben liabe, ist 6,0 . Als eben noch wirksam habe ich 2,0 pro die gefunden. Von 0,5 pro dosi und 1,0 pro die, wie der Erfinder will, habe ich absolut keinen Effect gesehen. Il dieser Weise habe ich 22 Fälle behandelt. Mit Erfolg 17, bei den übrigen war von der Therapie mit Alumen ustum entweder kein oder ein nur sehr geringer Erfolg zu verzeichnen.

Die einzelnen Krankengeschichten und Temperaturcurvelı ausführlich hier anzuführen, ist wohl überflüssig, da sie, was die 17 mit Erfolg behandeltell angeht, einen Fall ausgenommen, auf den ich später zurückkomme, alle die typische Febris intermittens ohne nennenswerthe Complicationen betreffen. Die Temperatur auf der Höhe des Anfalls schwankte zwischen 40 und 41, nur in 2 Fällen überstieg sie 41. Die Dauer des Fieberanfalls schwankte zwischen 2 und 8 Stunden. Bei allen diesen Fällen hatte die Therapie mit Alumen ustum den Erfolg, dass schon nach der einmaligen Darreichung des Medicamentes der erwartete Fieberanfall ausblieb, wenn sich auch die Zeit desselben durch ein leichtes Unbehagen seitens des Patienten markirte. Nur in einigen Fällen war nach der Darreichung noch eine Temperaturerhöhung zu constatiren, die aber gegen die des ersten Anfalls unverhältnissmässig gering und von viel kürzerer Dauer war. Auf den wiederholten Gebrauch des Alumen ustum in vergrösserter Dosis, das will sagen Vermehrung der einzelnen Dosen, kehrte ein Anfall nicht zurück.

Selbstredend liess ich nach dem Fortbleiben des ersten Anfalls Al. ust. noch 3 Tage lang in absteigender Dosis nehmen.

Die Krankengeschichte eines Falles verdient etwas mehr Interesse, sie sei deshalb kurz erwähnt.

Mingo, Jul., Füsilier, meldet sich krank, Temperatur 40,5. Milztumor, sonst objectiv nichts Abnormes zu constatiren, subjectiv Klagen über Kopfschmerzen und "Demam" (Fieber). Diaguose, mit Rücksicht auf den hier herrschenden Gellius endemicus, febris

\title{
V. Beiträge zur Behandlung der Febris intermittens mit Alumen ustum
} von F. Uhle,

pract. Arzt, Officier von gezondheid in der Niederländ. Ind. Armee, d. z. Garnisonsarzt auf Fort Rau, Sumatra.

Eine kurze Notiz im practiseerend geneeshoer" (der Name des Autors ist mir leider entfallen), die Alumen ustum als Surrogat für Chinin bei Febris intermittens empfahl, veranlasste mich, dieses Medicament auf den ihm vindicirten Werth zu prüfen.

Gelegenheit zu solchen Prüfungen sind in meiner Garnison in Fülle vorhanden, Rau gehört, was Malariainfectionen anlangt, zu den berüchtigtsten Garnisonen der Westküste Sumatras. Ueber welch reichhaltiges Material ich verfüge, erhellt wohl zur Genüge schon daraus, dass ich im Jahre 1884 bei einer Durchschnittsbesatzung von 50 Mann 360 Malariafälle behandelt habe, dabei habe ich 25 Fälle nicht mitgerechnet, die mich selbst betrafen.

Von den Fällen, die in meiner Privatpraxis zur Beobachtung kamen habe ich abgesehen, da sie aus äusseren Rücksichtell für derartige Beobachtungen ungeeignet sind, und einen Maassstab zur Beurtheilung des Klimas eben so wenig abgeben können.

Angewandt habe ich das Al. ust. bei dem reinen typischell Febr. interm., bei Febr. intermitt. mit Darmcomplicationen, bei auf Malariainfection beruhender Febris continua, und endlich bei einer Malariainfection, der ich den Namen Febris hectica geben möchte, ich rechne hierunter alle die Fälle, wo die Temperatur kaum über 38,5 sich erhebt, deutliche Remissionen oder Intermissionen nicht aufzuweisen hat, und wo es ausserordentlich schnell zu gefahrdrohendem Kräfteverfall, Kachexia paludosa kommt, und objectiv ausser einem Milztumor nichts zu constatiren ist. Ich will gleich bemerken, dass gegen diese Form der Malariainfection die einzig wirksame Therapie intermittens, zur Zeit der Untersuchung gerade ein Anfall. Am nächsten Tage zeigte sich, dass die Temperatur im grossen ulld ganzell sich auf derselben Höhe gehalten hatte, nur in den Morgenstunden voll 3-8 Uhr war sie bis 39 gefallen, war aber bei der Morgenvisite um 11 Uhr schon wieder im Steigen begriffen. Ich modificirte meine Diagnose dahin, dass ich die Affection für eine auf Malariainfection beruhende Febris continua hielt, da auch heute keine das Fieber erklärende Abnormität ausser dem Milztumor nachzuweisen war.

Ordo: Alumen ust. 3,0 in drei Dosen um 11, 12 und 1 Uhr. Abends Status idem wie gestern, Temperatur 40,5. Ordo: Alumen ustum 2,0, um 10 und $11 \mathrm{Uhr}$ Abends zu nehmen. Morgens $11 \mathrm{Uhr}$ fand ich, dass die Temperatur Morgens 6 Uhr 38 gewesen war, jetzt aber schon wieder 39,5 betrug. Ordo: Alum. ust. 6,0 in stündlichen Intervallen von je 1,0. Bei der Abendvisite Temperatur 36,5, die Temperatur hatte schon bei der 5. Dose angefangen zu fallell, NB. sie war bis 40,5 gestiegen und war dann sehr rapide zur Norm gefallen. Das Fieber recidivirte nicht wieder.

Was nun die kleinere Reihe von Fällen anlangt in denel Alum. ustum wirkungslos war, so fällt bei der Durchmusterung der Krankellgeschichten sofort auf, dass sämmtliche Fälle keine typisch reinen Malariafälle waren. In 2 Fällen war die Febris intermittens complicirt durch heftige Darmerscheinungen, 2 Fälle waren Febris contilua, und ein Fall war der Eingangs erwähnte Fall von Febris hectica.

In allen diesen Fällen griff ich, als Alum. ust. in Dosen zu 4,0 wirkunglos war, zum Chinin und fand, dass auch dieses nur ill grösseren Dosen 4,0-5,0 den gewünschten Effect hatte. Mit Ausnahme des letzten Falles, wo es ohne jeden Effect blieb, führte erst die Evacuation nach einem gesunden Garnisonsort die Genesung herbei.

Resümiren wir unsere Resultate, um zu einem Schluss zu kommen, so müssen wir uns wohl erst klar machen, warum man überhaupt bemüht ist, für das fast absolut sicher wirkende Chinin einen Ersatz zu finden. Wohl lediglich aus dreierlei Gründen, der theure 
Preis des Chinin, der schlechte Geschmack und die oft doch recht unangenehmen Nebenwirkungen. Ich habe Patienten behandelt, die Chinin in keiner Form vertrugen.

Der Preis des Alumen ustum ist ausserordentlich gering, aus diesem Grunde halte ich es für indicirt in der Armeepraxis. Der Geschmack ist indifferent, oder lässt sich sehr leicht verdecken, diese Eigenschaft macht es werthvoll für die Kinderpraxis. Unangenehme Nebenwirkungen des Alum. ust. selbst bei Dosen von 6,0 habe ich nicht zu verzeichnen gehabt. Ich würde das Alumen ustum demgemäss empfehlen, vorausgesetzt, dass es sich auch fernerhin bewährt, bei der typischen Febris intermittens, wenn äussere Gründe die Anwendung des Chinin schwierig oder unmöglich machen.

Halte ich die Versuche auch noch nicht für abgeschlossen, und bin ich mir recht wohl bewusst, dass 22 Fälle für den Werth eines Medicaments nicht entscheidend sind, so musste ich aus hier nicht näher zu erörternden Gründen schon jetzt mit der Publication der Versuche hervortreten, wenn ich sie überhaupt veröffentlichen wollte, und ieh glaube ihnen wenigstens das vindiciren zu können, dass sie geeignet sind, zu weiteren Versuchen aufzufordern. 